\title{
Heat and mass transfer in the hyperthermia cancer treatment by magnetic nanoparticles
}

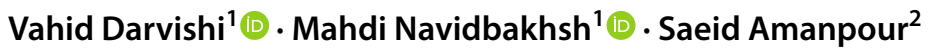

Received: 14 April 2021 / Accepted: 22 November 2021 / Published online: 26 November 2021

(c) The Author(s), under exclusive licence to Springer-Verlag GmbH Germany, part of Springer Nature 2021

\begin{abstract}
In this study, a more precise and cost-effective method is used for studying the drug delivery and distribution of magnetic nanoparticles in fluid hyperthermia cancer treatment, and numerical methods are employed to determine the effect of blood circulation on heat transfer and estimate the success of cancer treatment. A combination of numerical, analytical, and experimental researches is being conducted, which illustrates the essential role of numerical methods in medical and biomedical science. Magnetic NanoParticles' distribution and effects of infusion rate on the treatment are also discussed by considering the real distribution of MNPs. To increase accuracy and reduce costs in the in-vitro section, direct cutting and image processing methods are used instead of MRI. Based on the results of this section, with a tenfold increase in the infusion rate $(4 \mu \mathrm{l} /$ $\min$ to $40 \mu 1 / \mathrm{min}$ ), the penetration depth increases by $1 \mathrm{~mm}$, which represents a nearly 17 percent increase. Concentrations of MNPs also decrease significantly at higher infusion rates. The simulations of heat transfer reveal that maximum temperatures occur at the lowest infusion rate $(1.25 \mu \mathrm{l} / \mathrm{min})$, and blood flow also has a significant effect on heat transfer. With an increase in the infusion rate, necrosis tissue recedes from the tumor center and approaches the border between the tumor and healthy tissue. Results also show that, in lower MNPs' concentrations, higher infusion rates result in better treatment even though minimum infusion rates are suggested to be the best rates to facilitate distribution and treatment.
\end{abstract}

\section{Introduction}

Cancer is a disease that has high mortality rates, and there is no definitive treatment for it yet. Most of the cancer types appear in the form of a solid tumor. Cancerous cells can lead to cancer finally [1]. There are some prevalent treatments such as chemotherapy, radiotherapy and surgical ablation of cancerous tumors already [2] but all of them have many side effects, and they do not have enough accuracy $[3,4]$. In recent years, a new treatment called hyperthermia has been investigated widely and several experiments show its high efficacy while having fewer side effects [5-9]. Hyperthermia is a type of thermal treatment for cancer, in which

Vahid Darvishi

Darvishi.va@gmail.com

Mahdi Navidbakhsh

mnavid@iust.ac.ir

1 Tissue Engineering and Biological Systems Research Laboratory, School of Mechanical Engineering, Iran University of Science and Technology, 16846 Tehran, Iran

2 Cancer Biology Research Center, Tehran University of Medical Sciences, Tehran, Iran tumor cells are heated to a temperature above $42{ }^{\circ} \mathrm{C}$ and this leads to necrosis in living cells [10]. Increase in temperature also has been used for the diagnosis of the circulating cancer cells in blood [11]. There are many ways to raise the temperature of a tissue such as a laser, ultrasound and magnetic fluid. Laser irradiation as one of these methods was used successfully to make thermal damage, and the effects of some parameters such as the laser exposure time and the blood perfusion were considered [12-14]. Among all these methods, using magnetic fluid is the most effective way to raise the temperature in the body while has fewer side effects. In this method, a fluid containing magnetic nanoparticles (MNPs) is delivered into the tumor and generates adequate heating when exposed to the low alternating magnetic field. Killing tumor cells and making no side effects in healthy cells around the tumor is the primary purpose of this method. In magnetic fluid hyperthermia, the temperature can reach $42{ }^{\circ} \mathrm{C}$ to $45{ }^{\circ} \mathrm{C}$. Raising to $42{ }^{\circ} \mathrm{C}$ is mostly used to boost other methods of treatment, whereas raising over $45^{\circ} \mathrm{C}$ has been deployed as a cancer treatment method individually [15].

Heating the tumor cells leads to their necrosis or apoptosis in the magnetic fluid hyperthermia method $[16,17]$. 
Some parameters affect the results of killing cells in this method such as nanoparticle diameter, types of coatings of MNPs, aggregation of nanoparticles in magnetic fluid, and material used in the synthesis of MNPs.

Usov et al. worked on the relation between nanoparticle diameter and specific absorption rate. Results proved a substantial dependency [18]. An experimental trial indicated that cell-nanoparticle interaction reduces the cytotoxic effects of MNPs hyperthermia [19]. Dabbagh et al. suggested using a thermosensitive polymeric shell for coating the MNPs for obtaining a synergistic effect of hyperthermia. A 77\% decrease in cell viability due to the synergistic effects was observed using a new coating [20]. De Mello et al. worked on a series of zinc- and manganese-co-doped MNPs. The highest hyperthermia rate was reached by a manganeseonly doped and $\mathrm{Zn}$ doping decreased hyperthermia property [21]. Fabris et al. suggested a new method to control the heat generation of magnetic colloids by changing the shell composition of bimagnetic nanoparticles [22]. Engelmann et al. worked on the relation between the agglomeration of nanoparticles and heating efficiency. Results showed An increase of up to $23 \%$ for agglomerated MNPs in suspension and a decrease up to $28 \%$ for mixed states of immobilized and agglomerated MNPs [23]. Mai et al. showed it is possible to prevent the aggregation of the nanoparticle by simple irradiation of copper-mediated polymerization with ultraviolet light [24]. Esmaeili et al. suggested dendrimers as a material for MNPs synthesis because of their small size, well-defined globular shape and monodispersed. As a result of using dendrimers, superior heat generation was achieved [25]. Ota et al. worked on harmonic signals and measured them with respect to different MNPs structures to examine the key effects of particle structures on magnetization harmonics. A dependency between core size and anisotropy of MNPs and harmonics was observed in their work [26].

The distribution of MNPs in tissue after injection is another parameter that has effects on hyperthermia efficacy. As mentioned before, raising the temperature of tumor cells lead them to necrosis and it is highly related to the position of MNPs after the injection, so it is vital to know about MNPs distribution and concentration after injection. For a long time, MNPs were believed to be distributed uniformly after injection, but it is now been established that the concentration is not uniform after injection [2, 27, 28]. There are some parameters that can cause a non-uniform distribution.

Chen et al. investigated the effects of changing the rate of the infusion, cannula size, and the concentration of infusate on the fluid dispersion after injection. They found that higher infusion rates can cause more leak back of the infusate than lower infusion rates. It was also claimed that varying the concentration of the infusate does not affect the volume of the distribution [29]. Salloum et al. examined some parameters and their effects on MNPs distribution such as infusion flow rate and agarose gel concentration. It was concluded that the infusion flow rate and concentration could affect the distribution of MNPs. By decreasing the infusion rate, distribution becomes more spherically shaped [28]. Navidbakhsh et al. also investigated the effect of infusion rate on MNPs distribution with the help of computer simulation in an agarose gel. They developed a model for temperature distribution [30]. Kandala et al. used six different MNPs distributions to simulate heat transfer in tissue during hyperthermia by magnetic fluid. It was concluded that in concentrated nanoparticles distribution, modulated power heating makes less damage on surrounding normal tissue compared to constant power heating [31]. Gu et al. examined effects of heating on MNPs concentration distribution in prostatic cancer tumors. It was found that MNPs distribution volume in high heat generation rate was 10 percent smaller in the heating group, while in low heat generation rate it was 95 percent larger in the heating group [32].

In the hyperthermia cancer treatment by MNPs, several parameters may affect the results of treatment such as infusion rate, nanoparticle concentration in the magnetic fluid, nanoparticles diameter, coating type and surface charge of nanoparticles, type of the tissue and type of the needle used for injection and also blood flow. Among these parameters, the infusion rate has more effects, and it is also controllable. Moreover, blood flow can play a vital role in heat transfer during the treatment. Since the thermocouples had errors while a magnetic field was applying, simulation of heat transfer could help scientists to determine. Different investigations showed the effectiveness of using numerical methods for the estimation of the temperature [33-35].

\section{Materials and methods}

\subsection{In-vitro}

To investigate the effects of infusion rate on distribution and as a consequence on hyperthermia cancer treatment, a fluid Hamilton syringe with a maximum reservoir volume of 250 $\mu l$ was used to inject fluid into the tissue. A 26-gauge Hamilton needle was also used to avoid the backflow of magnetic fluid. For injecting MNPs into the tissue at a constant rate, a syringe pump was needed. The only available pump was the Te-331 syringe pump with the retaining clip that only was able to handle large syringes with a size between 10 and $50 \mathrm{ml}$, so a $50 \mathrm{ml}$ syringe was prepared and the Hamilton syringe was fixed inside it to be put into the syringe pump.

Subsequently, since input numbers for the pump showed the infusion rate and volume of injections per the $50 \mathrm{ml}$ syringe, a conversion between the $50 \mathrm{ml}$ syringe and the fixed Hamilton syringe must be obtained, which was 
Fig. 1 Length and width used to measure the distribution

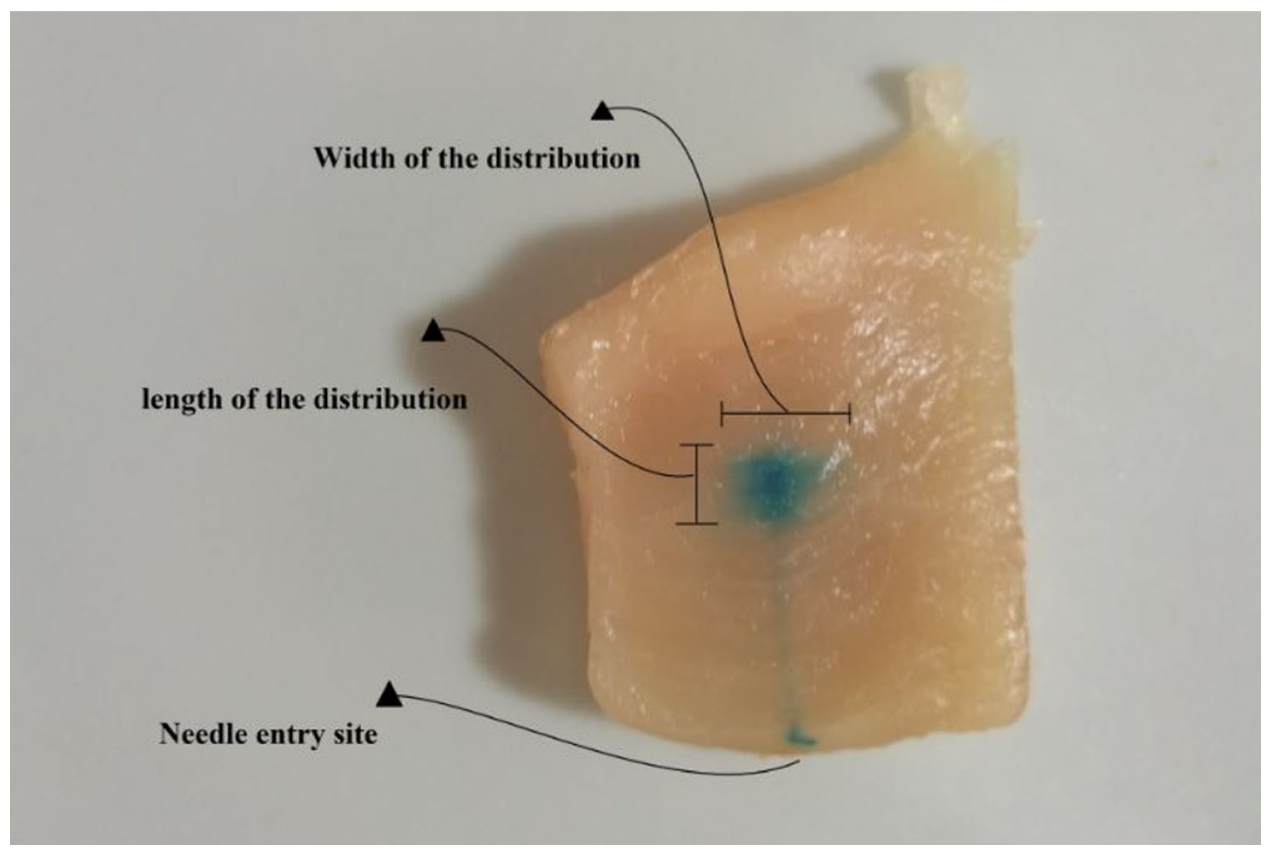

achieved by taking some tests and determining the time of each test. By performing these experiments, the ratio of 1 to 160 was obtained and formulated as Eq. 1 for the pump infusion rate and the actual rate of Hamilton syringe injection.

$\alpha V_{H}=V_{P}$

In Eq. (1), $\alpha=9.6$ and $V_{H}$ and $V_{P}$ denote the input velocity for the Hamilton syringe $(\mu \mathrm{l} / \mathrm{min})$ and input velocity for the infusion pump ( $\mathrm{ml} /$ hour), respectively.

$\mathrm{MoS}_{2} / \mathrm{CoF}_{2} \mathrm{O}_{4}$ that had been examined and used in previous in-vivo tests was prepared and considered as magnetic fluid. $\mathrm{MoS}_{2}$ had semi-conductor properties in addition to a large surface area, and introduction of $\mathrm{CoF}_{2} \mathrm{O}_{4}$ on the surface of $\mathrm{MoS}_{2}$ led to create hetero-structures. Results of previous investigations showed an improvement in electromagnetic absorptions of hybrids of $\mathrm{MoS}_{2}$ and $\mathrm{CoF}_{2} \mathrm{O}_{4}$ [36]. Moreover, no sign of inflammation or necrosis in mice liver was reported in the injection of $\mathrm{MoS}_{2} / \mathrm{CoF}_{2} \mathrm{O}_{4}$. The size of MNPs was also an important parameter in hyperthermia since it affected the specific loss power (SLP) [37]. MNPs with the size of $17 \pm 4 \mathrm{~nm}$ was considered since it could provide a sufficient SLP for hyperthermia treatment and was dispersed in aqueous very well [36].

The chicken breast tissue was used in this study because of its availability, low cost and brightness. The brightness of tissue was important since this brightness made it possible to determine the distribution of a substance after injection by using image processing techniques. Using chicken tissue for in-vitro investigation of cancer was reported before [38-40].

In fluid injection, 14 different slices were selected for testing various infusion rates between $1.25 \frac{\mathrm{\mu l}}{\mathrm{min}}$ and $104.06 \frac{\mathrm{\mu l}}{\mathrm{min}}$ (Maximum infusion rate produced by the infusion pump). To have comparable results with previous experimental works, a range of $10 \frac{\mu l}{\mathrm{~min}}$ to $40 \frac{\mathrm{\mu l}}{\mathrm{min}}$ was considered for infusion rate. Each infusion rate was repeated three times at best because of limitation of the available nanofluid.
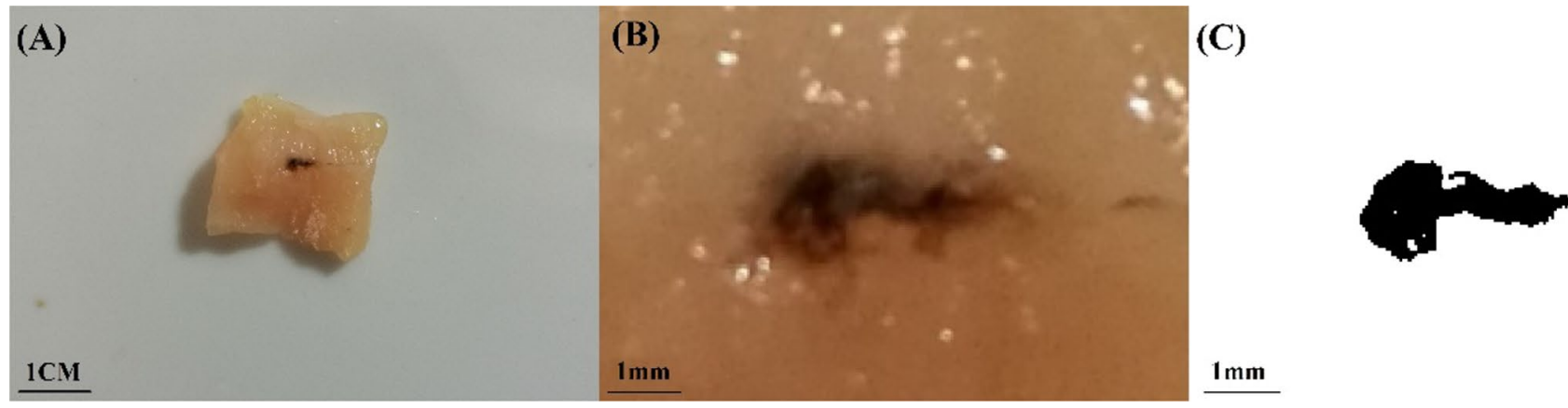

Fig. 2 (A) The original image. (B) Image after cutting into a $9 \mathrm{~mm} * 6 \mathrm{~mm}$ image. (C) Image after image processing 
For cutting the tissue, a standard surgical blade was used. The cutting section was considered perpendicular to the injection site as shown in Fig. 1. The crucial issue in this part was the probability of human errors in the cutting of the tissue that could be eliminated by using micron cutting devices. Access to these kinds of devices had been confronted with limitations. Therefore, the number of tests was considered 3 in each case, to minimize human errors in the cutting section.

A Canon PC1680 camera was used to take photos, under a standard light condition and in the absence of sunlight.

\subsection{Image processing}

In the next step, all images were prepared for the image processing section. The preparation process began with cutting the part of the texture image off. Since the real tumors had an oval shape with a large diameter of $9 \mathrm{~mm}$ and a small diameter of $6 \mathrm{~mm}$ [36], in the image processing section, a part of the original image which its dimensions were $2 \mathrm{~cm} * 2 \mathrm{~cm}$, was cut to a rectangle of $9 \mathrm{~mm} * 6 \mathrm{~mm}$ to make the results of this study comparable with previous in-vivo works. A Schematic of these steps is illustrated in Fig. 2.

The MATLAB software was deployed for image processing in this study. To achieve the distribution of MNPs, all color images were converted into black-and-white images. In the black-and-white pictures, a threshold between 0 and 1 was determined for the MATLAB software to delete points of images that were brighter to separate darker points as nanoparticles position. By extracting the coordinates of all black pixels, the distribution of MNPs was obtained.

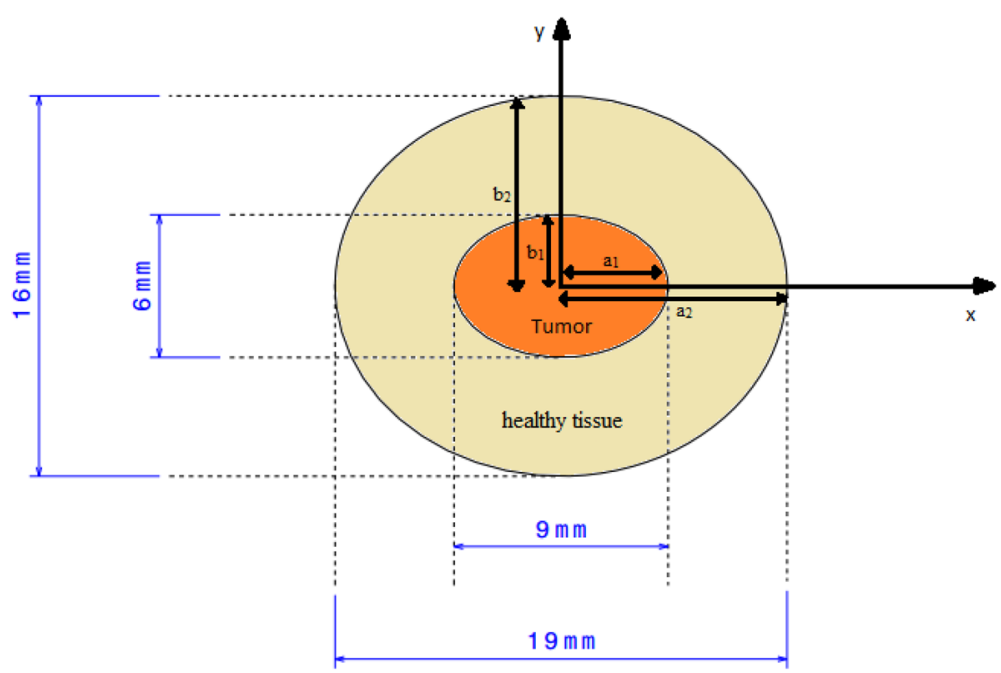

(A)
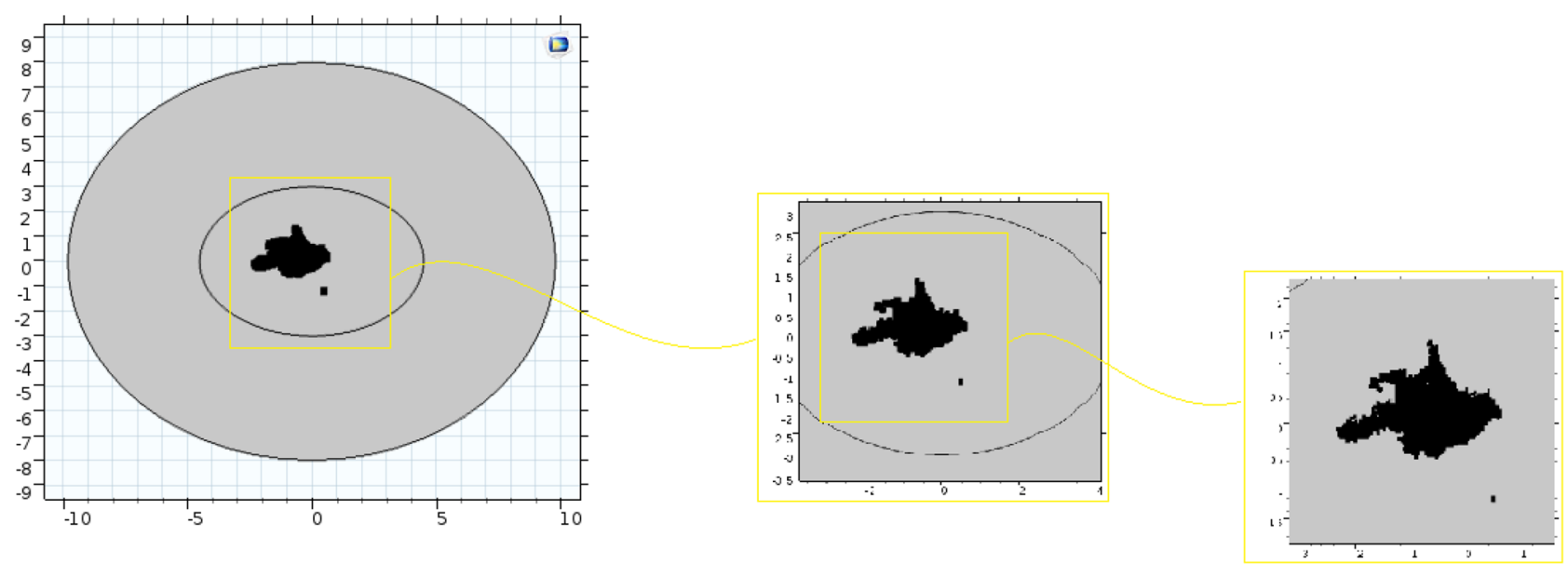

(B)

Fig. 3 (A) The geometry of the simulated tumor and the surrounding healthy tissue [41]. (B) The geometry after adding nanoparticles 


\subsection{Simulation of heat transfer}

Thermocouples had errors while a magnetic field was applying. Due to limitations in measuring the temperature in an experimental trial, simulation of heat transfer was deployed to determine the temperature of any point in the tissue which was warmed by nanoparticles. The role of blood flow on heat transfer was also investigated using numerical methods.

\subsubsection{Geometry}

A two-dimensional geometry was considered to make the simulation results comparable with future laboratory and animal works for the tumor. Based on previous animal works, an ellipse with a large diameter of $9 \mathrm{~mm}$ and a small diameter of $6 \mathrm{~mm}$ was created in COMSOL software using geometry tools. Around this ellipse, another ellipse with a large diameter of $19 \mathrm{~mm}$ and a small diameter of $16 \mathrm{~mm}$ was considered to represent the healthy tissue surrounding the tumor. The geometry is schematically illustrated in Fig. 3A.

After creating the desired geometries, coordinates of points obtained by the MATLAB software as the distribution of MNPs were imported into the COMSOL software and added to the geometry. An example of the geometry after adding points as nanoparticles is illustrated in Fig. 3B.

\subsubsection{Material}

Properties of materials used in this simulation are presented in Table 1. Although there was no blood in the chicken breast tissue, blood properties were also added in the model (Table 2). The reason for considering the properties of the blood was to investigate its effect on the heat transfer and also made the numerical model more realistic.

Table 1 Mechanical properties of the healthy and tumor tissue used in simulation [42-44]

\begin{tabular}{llll}
\hline Property & Symbol & \multicolumn{2}{l}{ Tissue type } \\
\cline { 3 - 4 } & & Tumor & Healthy \\
\hline Specific Heat $\left(\frac{\mathrm{J}}{\mathrm{kg} \cdot \mathrm{K}}\right)$ & $C$ & 3760 & 3760 \\
Density $\left(\frac{\mathrm{kg}}{\mathrm{m}^{3}}\right)$ & $\rho$ & 1045 & 1045 \\
Thermal Conductivity $\left(\frac{\mathrm{W}}{\mathrm{m} \cdot \mathrm{K}}\right)$ & $k$ & 0.51 & 0.51 \\
Pre-factor $\left(\frac{1}{s}\right)$ & $A$ & $1.80 \mathrm{E} 36$ & $1.03 \mathrm{E} 38$ \\
Activation Energy $\left(\frac{J}{m o l}\right)$ & $E_{a}$ & $2.38 \mathrm{E} 5$ & $2.49 \mathrm{E} 5$ \\
Point Heat Source $\left(\frac{\mathrm{W}}{\mathrm{m}}\right)$ & $Q_{p}$ & 0.016 & 0 \\
Perfusion Rate $\left(\frac{1}{\mathrm{~s}}\right)$ & $\omega$ & 0.0095 & 0.003 \\
Metabolic Heat Rate $\left(\frac{\mathrm{W}}{\mathrm{m}^{3}}\right)$ & $Q_{m}$ & $31,872.5$ & 6374.5 \\
\hline
\end{tabular}

Table 2 Mechanical properties of the blood used in simulation[42, 44]

\begin{tabular}{lll}
\hline Property & Symbol & blood \\
\hline Specific Heat $\left(\frac{\mathrm{J}}{\mathrm{kg} \cdot \mathrm{K}}\right)$ & $C_{b}$ & 3770 \\
Density $\left(\frac{\mathrm{kg}}{\mathrm{m}^{3}}\right)$ & $\rho_{b}$ & 1060 \\
Temperature $\left({ }^{\circ} \mathrm{C}\right)$ & $T_{b}$ & 37 \\
\hline
\end{tabular}

The point heat source was considered $0.016 \frac{\mathrm{W}}{\mathrm{m}}$. This consideration was based on having a maximum temperature of about $46{ }^{\circ} \mathrm{C}$ in the uniform distribution which was a standard value in clinical studies [7].

\subsubsection{Governing equations}

In the simulation of physics of the model, Pennes' bio-heat equation, as illustrated in Eq. (2), was considered as the governing equation. The reason was the correspondence of this equation with experimental studies [45].

$\rho \complement \frac{d T(x, y, t)}{d t}=k_{t} \nabla^{2} T(x, y, t)-\rho_{b} \complement_{b} \omega_{b}\left(T(x, y, t)-T_{b}\right)+Q_{m}+Q_{p}$

In Eq. (2), $\rho, \complement, T, k$ denote the tumor/healthy tissue density, tumor/healthy tissue specific heat, local tumor/healthy tissue temperature and thermal conductivity of the tumor/ healthy tissue, respectively. $\mathrm{t}$ denotes time and $\rho_{b}, \complement_{b}, \omega_{b}, T_{b}$ denote blood density, specific heat of blood, blood perfusion rate and blood temperature, respectively. $Q_{m}$ denotes the metabolic heat generation rate in the tumor/healthy tissue and $Q_{p}$ denotes the point heat source in the tumor/healthy tissue.

The Arrhenius equation was also deployed in the simulation section. This equation indicates the amount of necrotized tissue after a certain period of time and is expressed in Eq. (3) [46]:

$\Omega(x, y, t)=A \int_{0}^{t} e^{-E_{a} / R_{u} T(x, y, t)} d_{t}$

In Eq. 3, $A, E_{a}, R_{u}$ denote pre-exponential factor, activation energy, and universal gas constant, respectively.

\subsubsection{Boundary and initial conditions}

For boundary conditions, the continuity of temperature and heat flux in the boundary between tumor and healthy tissue was added to the model (Eq. (4) and Eq. (5)):

$T_{t}(x, y, t)=T_{h}(x, y, t), \frac{x^{2}}{a_{1}^{2}}+\frac{y^{2}}{b_{1}^{2}}=1$ 
$k_{t} \frac{\partial T_{t}(x, y, t)}{\partial n}=k_{h} \frac{\partial T_{t}(x, y, t)}{\partial n}$

Which indicated the equivalence of temperature and flow flux in the boundary between the tumor and the healthy tissue.

The second boundary condition was related to the outer surface temperature of the healthy tissue, which was constant and equal to body temperature or $37^{\circ} \mathrm{C}$. This boundary condition can be expressed as follows:

$T_{h}(x, y, t)=37^{\circ} \mathrm{C}, \frac{x^{2}}{a_{2}^{2}}+\frac{y^{2}}{b_{2}^{2}}=1$

The only initial condition that had been used in this study was the initial temperature of the healthy tissue and tumor, which was considered $37^{\circ} \mathrm{C}$ and can be expressed as follows:

$T(x, y, 0)=37^{\circ} \mathrm{C}$

In Eq. (4) to Eq. (7), $a_{1}=4.5 \mathrm{~mm}, a_{2}=9.5 \mathrm{~mm}, b_{1}=3 \mathrm{~mm}$ and $b_{2}=8 \mathrm{~mm}$.

The bio-heat transfer equation could be summarized and solved using finite element methods (FEM). The finite element technique was a powerful method in numerical solutions that could solve Eq. (2) with boundary and initial conditions Eq. (4) to Eq. (7). FEM divided a complex system into smaller parts called finite elements to solve a problem. This could be achieved by implementing space discretization, which was accomplished by building a mesh for the model. An algebraic equation system could be ultimately derived from the finite element method formulation of a boundary value problem. These equations were then grouped into a larger system that fit the whole model. Using the calculus of variations, the FEM approximated a solution by minimizing a function of errors.

\subsubsection{Time step and mesh configuration}

The overall time of $1200 \mathrm{~s}$ was considered in simulations since laboratory animals used in clinical trials could be anesthetized and treated up to $20 \mathrm{~min}$. The Timestep of $1 \mathrm{~s}$ was also considered to have the temperature and the percentage of necrosis tissue in each second. A triangular mesh was used as a mesh type and generated by tools provided in COMSOL software. Each triangle had three sides with a specific magnitude. The maximum and minimum magnitude was considered for element's (sides of triangles) size in
Table 3 Comparison of maximum temperature for different mesh types

\begin{tabular}{lllll}
\hline Mesh type & \multicolumn{2}{l}{ Element size $(\mathrm{mm})$} & $\begin{array}{l}\text { Total number } \\
\text { of elements }\end{array}$ & $\begin{array}{l}\text { Maximum } \\
\text { temperature } \\
\left({ }^{\circ} \mathrm{C}\right)\end{array}$ \\
\cline { 2 - 5 } & Max & Min & & 51.28088 \\
Normal & 1.27 & 0.0057 & 5666 & 51.2809 \\
Fine & 1.01 & 0.0057 & 5884 & 51.28094 \\
Finer & 0.703 & 0.00238 & 7026 & 51.28519 \\
Extra Fine & 0.38 & 0.00142 & 17,347 & 51.28505 \\
Extremely Fine & 0.19 & $3.8 \mathrm{E}-4$ & 33,276 & \\
\hline
\end{tabular}

different mesh types to control the total number of triangles and as a result the resolution of the final results. To ensure the results were not affected by the time step and mesh size and were accurate, the maximum temperature was calculated for different mesh sizes at a constant time step of $1 \mathrm{~s}$. Results represented an error of about $0.005^{\circ} \mathrm{C}$ that could be ignored. The maximum temperature was also calculated for different time steps at constant normal mesh size. In this section, no error was observed. Results of this section which are illustrated in Tables 3 and 4, showed the independence of the results from the mesh size and the time step. Considering the results of this section, the time step of $1 \mathrm{~s}$ and normal mesh type were chosen for simulation.

\subsection{Validation of simulation}

Numerical models could be validated by comparing their results with an experimental or analytical investigation. This was a common method to validate a numerical model and authors had used it in other numerical researches such as coronavirus area too [47]. It was vital to have the same properties, initial and boundary conditions and set up to be able to compare the results. The numerical model used in this study was verified against the results of Lin and Liu [48], for the radial distribution of temperature in the tissue with the same tissue property, initial, and boundary condition. The model was developed then with an elliptical shape instead of the circular but with the same initial and boundary conditions as Lin and Liu's model. A comparison of the simulation with the results of Lin and Liu is illustrated in Fig. 4. In addition to verifying the numerical model with an analytical investigation, independence of the model from time step and mesh size was also checked in the previous section to be sure about the validation of the results.
Table 4 Comparison of maximum temperature for different time steps

\begin{tabular}{llllll}
\hline Time step $(\mathrm{s})$ & 0.1 & 0.5 & 1 & 2 & 10 \\
\hline Maximum temperature $\left({ }^{\circ} \mathrm{C}\right)$ & 51.28088 & 51.28088 & 51.28088 & 51.28088 & 51.28088 \\
\hline
\end{tabular}


Fig. 4 Radial distribution of temperature in the tissue after $600 \mathrm{~s}$, a comparison between present work and Lin and Liu's [48]

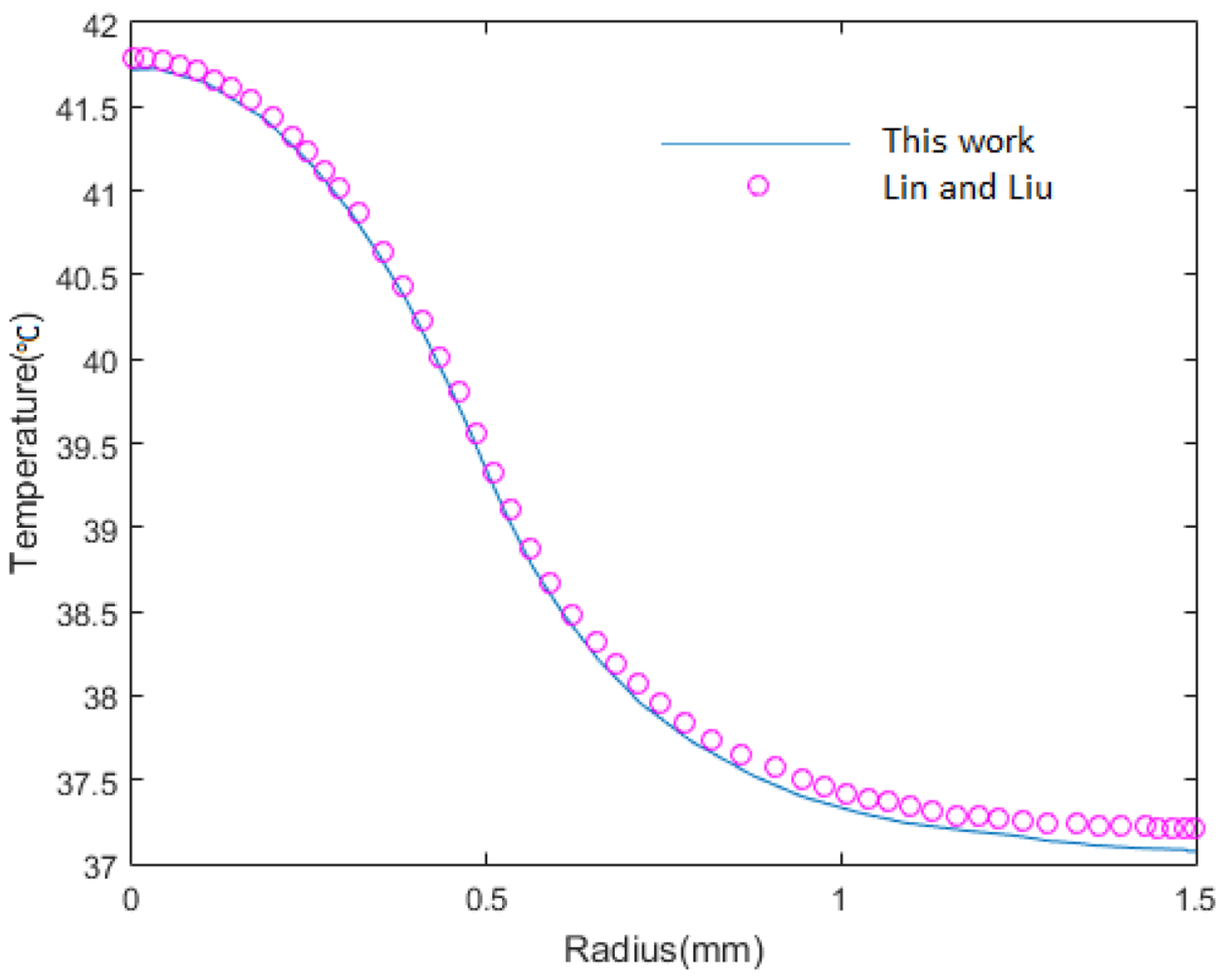

\section{Results and discussion}

\subsection{Infusion rates of MNPs}

Increasing the infusion rate leads to an increase in nanoparticle penetration in tissue. Results show congruence with the results of Attaluri's work [49]. However, the notable point is the magnitude of the increase. According to Attaluri's work, with an increase in the infusion rate from $5 \frac{\mu l}{\mathrm{~min}}$ to $10 \frac{\mu l}{\min }$, an increase of $50 \%$ in penetration depth occurs, while in current experiments, with an increase in the infusion rate from $4 \frac{\mu l}{\min }$ to $40 \frac{\mu l}{\min }$ or in other words, with ten

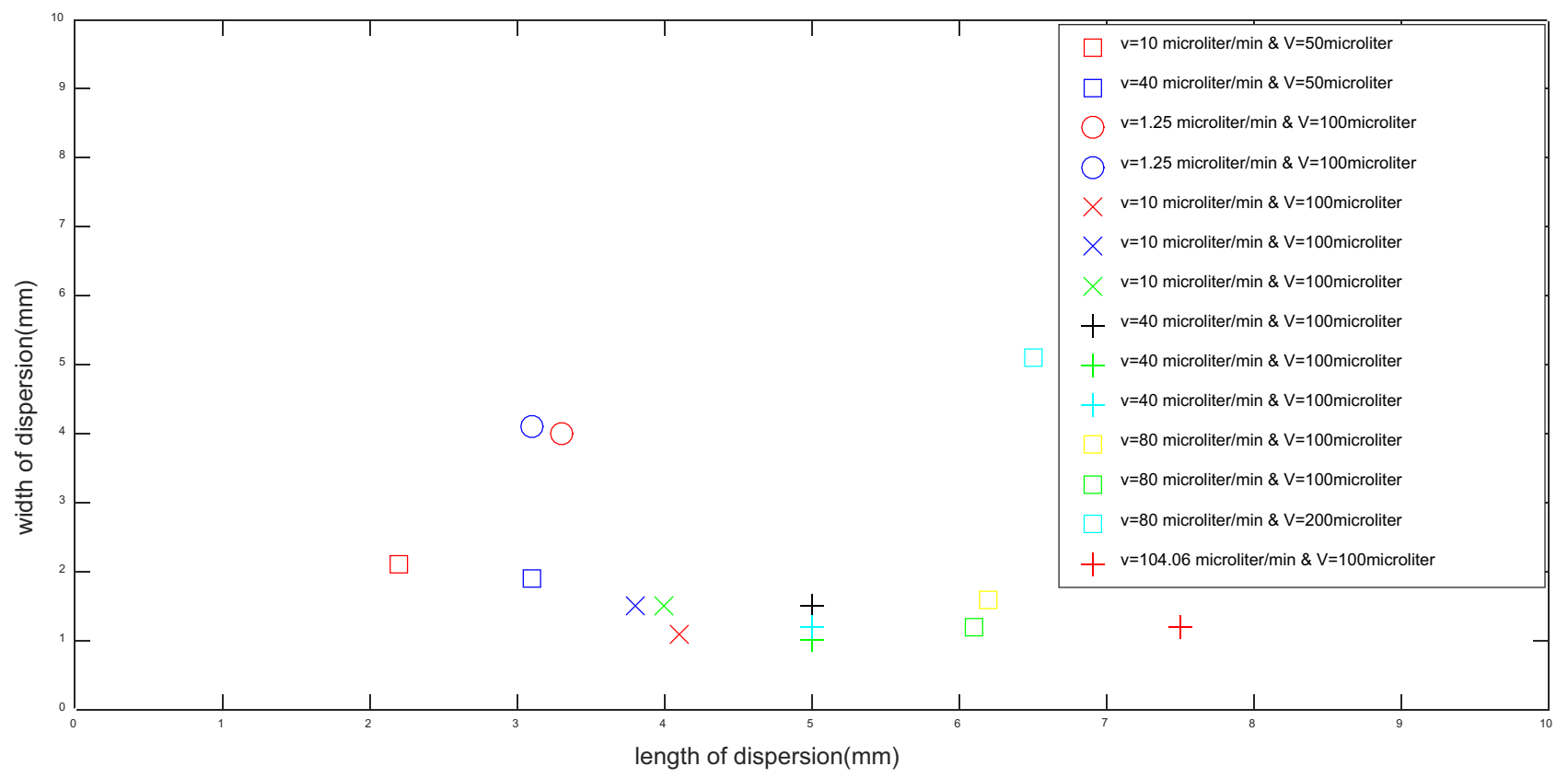

Fig. 5 Distribution length and width in MNPs injection with different concentrations and infusion rates 
Fig. 6 Concentration of MNPs in the tissue. (A) Injection of $100 \mu l$ of magnetic fluid with an infusion rate of $1.25 \frac{\mu l}{m i n}$. (B) Injection of $100 \mu l$ of magnetic fluid with an infusion rate of $104.06 \frac{\mu l}{\min }$

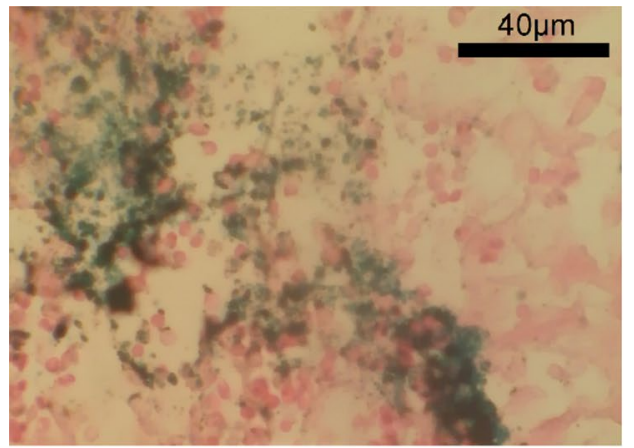

(A)

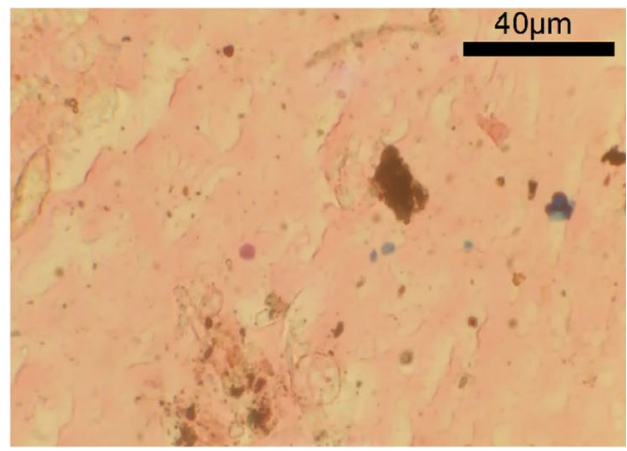

(B) times the infusion rate, there is a $1-\mathrm{mm}$ increase in penetration depth, which represents a nearly 17 percent increase in penetration depth. The reason may be the type of tissue tested and the difference in the tests performed with the 2011 Attaluri's work. Another reason could be the use of different procedures to examine the distribution of MNPs since Attaluri used CT scan imaging techniques, while in the present study, cutting and direct imaging method has been used. As shown in Fig. 5, the best distribution occurs in the lowest infusion rate of $1.25 \frac{\mu l}{\min }$, which is almost circularly distributed and has similarity with the results of 2008 Salloum's work on the agarose gel.

\subsection{The concentration of MNPs for different infusion rates}

Comparing different samples under the microscope reveals a lower concentration of MNPs could be achieved at higher infusion rates. This decrease in concentration could happen since, in higher infusion rates, nanoparticles are distributed in a bigger area. The effects of infusion rate on MNPs concentration are illustrated in Fig. 6.

\subsection{Simulation of heat transfer in nanoparticle testing at different infusion rates}

By having the results obtained in the simulation of heat transfer section, and considering the gradual increase in penetration depth by increasing the infusion rate and consequently excretion of nanoparticles from the border of the tumor and healthy tissue, it is determined that the tissue necrosis happens in healthy tissue at higher rates. This necrosis of healthy tissue occurs while some parts of the tumor do not feel any increase in temperature and consequently will not be necrosis at all. Therefore, it could be concluded that higher infusion rates should be avoided to have a circular shape distribution and also to prevent the penetration of nanoparticles into healthy tissue. By obtaining the specific distribution of magnetic nanoparticles in the tissue, the maximum temperature in different cases is higher than the maximum temperature in a uniform distribution (less than $46^{\circ} \mathrm{C}$ ). This difference indicates the impact of the distribution of MNPs in tissues on the maximum produced temperature.

Table 5 The maximum produced temperature for different injection volumes and infusion rates

\begin{tabular}{|c|c|c|c|c|c|c|c|c|c|c|}
\hline \multirow[t]{3}{*}{ Test number } & \multirow{3}{*}{$\begin{array}{l}\text { Injection volume \& } \\
\text { infusion rate }\end{array}$} & \multicolumn{9}{|c|}{ Maximum produced temperature for sample... $\left({ }^{\circ} \mathrm{C}\right)$} \\
\hline & & \multicolumn{3}{|c|}{$\begin{array}{l}\text { No blood in both healthy and } \\
\text { tumor tissue }\end{array}$} & \multicolumn{3}{|c|}{ Having blood just in healthy tissue } & \multicolumn{3}{|c|}{$\begin{array}{l}\text { Having blood in both healthy } \\
\text { and tumor tissue }\end{array}$} \\
\hline & & 1 & 2 & 3 & 1 & 2 & 3 & 1 & 2 & 3 \\
\hline 1 & $50 \mu l \& 10 \frac{\mu l}{\min }$ & 48.7923 & - & - & 48.3825 & - & - & 46.005 & - & - \\
\hline 2 & $50 \mu l \& 40 \frac{\mu l}{\min }$ & 47.8011 & - & - & 47.3684 & - & - & 45.093 & - & - \\
\hline 3 & $100 \mu l \& 1.25 \frac{\mu l}{\min }$ & 57.2387 & 56.4372 & - & 56.4382 & 55.6637 & & 51.985 & 51.562 & - \\
\hline 4 & $100 \mu l \& 10 \frac{\mu l}{\mathrm{~min}}$ & 55.8848 & 56.0444 & 57.0162 & 55.0797 & 55.2389 & 56.2428 & 51.033 & 51.280 & 52.006 \\
\hline 5 & $100 \mu l \& 40 \frac{\mu l}{\min }$ & 54.0376 & 55.9015 & 55.9163 & 53.2398 & 55.1175 & 55.1257 & 49.189 & 50.931 & 50.953 \\
\hline 6 & $100 \mu l \& 80 \frac{\mu l}{\mathrm{~min}}$ & 54.4726 & 54.1680 & - & 53.7006 & 53.3390 & - & 49.636 & 49.535 & - \\
\hline 7 & $200 \mu l \& 80 \frac{\mu l}{\min }$ & 70.6874 & - & - & 69.1438 & - & - & 64.360 & - & - \\
\hline 8 & $100 \mu l \& 104.06 \frac{\mu l}{\mathrm{~min}}$ & 52.5343 & - & - & 51.7507 & - & - & 48.216 & - & - \\
\hline
\end{tabular}




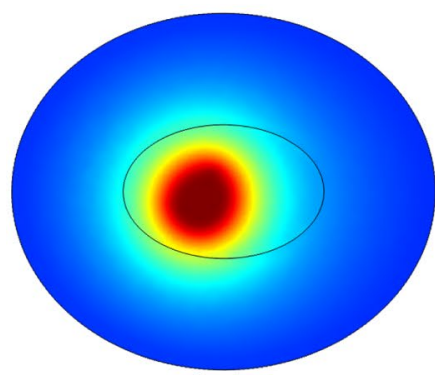

(A)

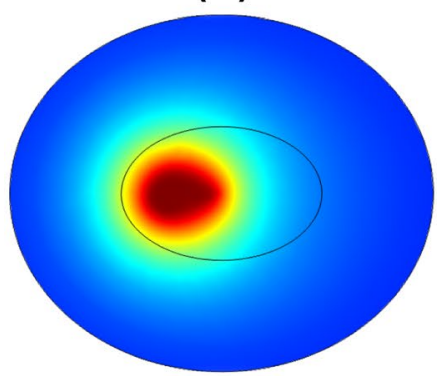

(B)

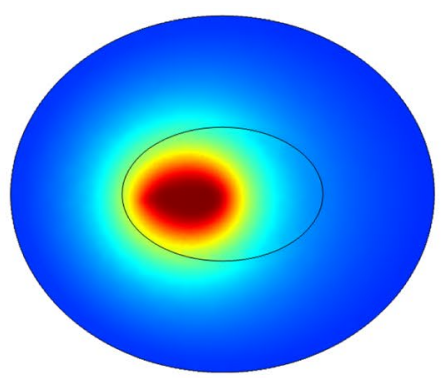

(C)

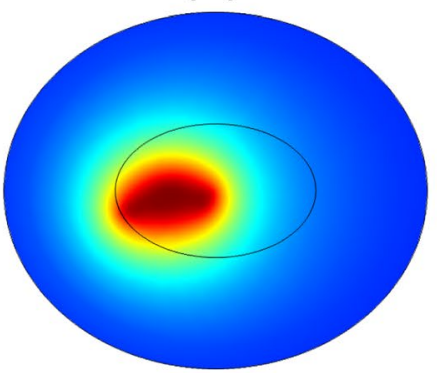

(D)

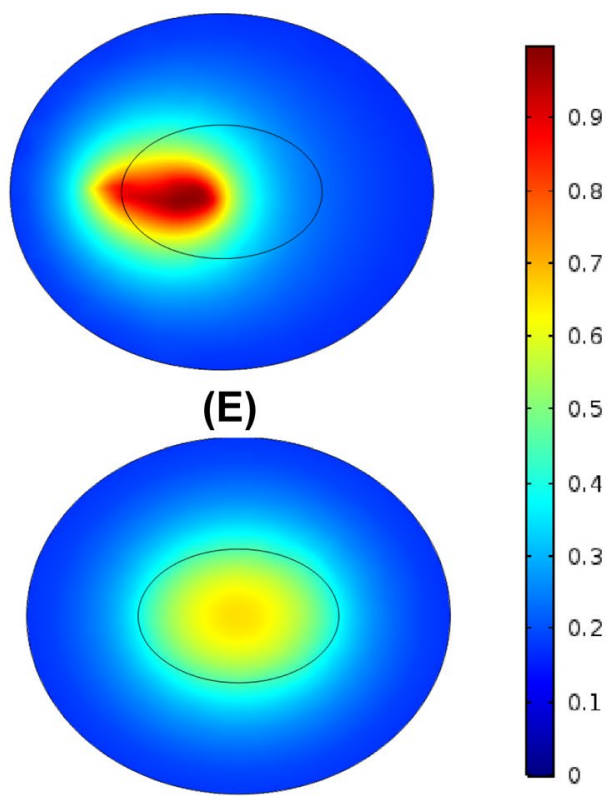

(F)

Fig. 7 The fraction of necrotic tissue after $1200 \mathrm{~s}$. (A) Infusion rate $=1.25 \frac{\mu l}{\mathrm{~min}}$. (B) Infusion rate $=10 \frac{\mu l}{\mathrm{~min}}$. (C) Infusion rate $=40 \frac{\mu l}{\mathrm{~min}}$. (D) Infusion rate $=80 \frac{\mu l}{\min }$. (E) Infusion rate $=104.06 \frac{\mu l}{\min }$. (F) Uniform distribution (control model)

Due to the maximum temperatures obtained in each section, it can not be concluded that there is a correlation between the infusion rate and the maximum temperature. As shown in Table 5, for an infusion rate of $1.25 \frac{\mathrm{ml}}{\mathrm{min}}$, the maximum temperature is about $51.985^{\circ} \mathrm{C}$, which by increasing the infusion rate up to $10 \frac{\mathrm{ml}}{\mathrm{min}}$, reaches about $51.033^{\circ} \mathrm{C}$. The reason can be reducing the distribution area, or in other words, concentration of nanoparticles in a smaller area which is consistent with the results of the experimental section (Concentration of MNPs for different infusion rates). The lack of uniform distribution of nanoparticles also affects the magnitude of maximum temperature at a specific point. For instance, in Sample 1 of the fifth test, the maximum temperature is $49.189^{\circ} \mathrm{C}$, while in the similar sample of the same test (Sample 3 of the fifth test), the maximum temperature is about $50.953{ }^{\circ} \mathrm{C}$, which is about $1.8^{\circ} \mathrm{C}$ of difference. Also, other reasons such as the impact of the image processing and the type of cutting and photographing may also affect the results in each section, but in general, and for samples after the fourth test, which the distribution width is almost constant, it shows a very slight decrease in maximum temperature that is similar to 2011 Attaluri results. In other words, maximum temperature occurs in minimum infusion rates $\left(1.25 \frac{\mu l}{\min }\right)$.

Another noteworthy point obtained from the results is the role of blood flow in heat transfer. As it is shown in Table 5, blood has a significant effect on heat transfer in tissue, and as a result, magnetic fluid hyperthermia cancer treatment. Maximum temperature can be increased up to
$9{ }^{\circ} \mathrm{C}$ (test8) in the absence of blood flow in the tissue. Results of the effects of blood on heat transfer are divided into three sections. Having no blood in both healthy and tumor tissue, having blood just in healthy tissue, and finally having blood in both healthy and tumor tissue.

The last point to be taken from the temperature distribution in the tissue is the maximum temperature point, which by increasing the infusion rate, recedes from the tumor center (which is the injection site) and approaches the borders of the tumor and healthy tissue (Fig. 7.).

\section{Conclusion}

Cancer is one of the most vital causes of death in recent years, and several methods have been suggested for treating it. Hyperthermia is one of the emerging methods of treatment that is more accurate and has fewer side effects compared to other treatments. Although many investigations have been conducted in this area, there is a lack of knowledge in some parameters such as the role of blood flow on MNPs hyperthermia, diffusion of MNPs, temperature distribution during treatment, and tumor necrosis after treatment yet. In this study, a different technique is used to measure the depth of penetration compared to previous works and a more precise ratio between penetration depth and infusion rate is achieved as a result. The results of experiments on MNPs show that by increasing the infusion rate, the penetration depth increases. With an increase in 
the infusion from $4 \frac{\mu l}{\min }$ to $40 \frac{\mu l}{\min }$, there is a $1-\mathrm{mm}$ increase in penetration depth (nearly 17 percent increase). This increase in infusion rate also has a significant impact on the concentration of MNPs in tissue. Increasing the infusion rates leads to a lower concentration of MNPs.

The numerical simulation reveals that blood flow in tumor and healthy tissue plays an important role in heat transfer in the tissue (in addition to mass transfer of MNPs out of tumor area made by blood flow, which has been investigated in previous investigations). The numerical simulation also showed that there is not a clear and precise relationship between increasing the penetration depth and the maximum temperature created in the tissue, but it can be concluded that by increasing the infusion rate and consequently increasing penetration depth, the maximum temperature decreases slightly which means maximum temperature occurs by the infusion rate of $1.25 \frac{\mathrm{ml}}{\mathrm{min}}$.

Finally, the results of the necrosis section reveal that by increasing the infusion rate, necrosis has occurred in healthy tissue rather than cancerous tissue, which should be avoided by reducing the infusion rate. Moreover, the location of the necrosis recedes from the tumor center and approaches the borders of the tumor and healthy tissues by increasing the infusion rate.

\section{Authors' contributions Not applicable.}

Funding The authors did not receive support from any organization for the submitted work.

Data availability Data are easily accessible in any time.

Code availability Codes are available at any time.

\section{Declarations}

Ethics approval Not applicable.

Consent to participate The authors agree to participate.

Consent for publication The authors agree to publication this article.

Conflicts of interest The authors have no conflicts of interest to declare that are relevant to the content of this article.

\section{References}

1. Hanahan D, Weinberg RA (2000) The hallmarks of cancer. Cell 100:57-70

2. Attaluri A, Ma R, Qiu Y et al (2011) Nanoparticle distribution and temperature elevations in prostatic tumours in mice during magnetic nanoparticle hyperthermia. Int J Hyperth 27:491-502. https://doi.org/10.3109/02656736.2011.584856
3. Truskey GA, Yuan F, Katz DF (2004) Transport phenomena in biological systems

4. National Cancer Institute. http://www.cancer.gov/cancertopics/ treatment/types-of-treatment

5. Jordan A, Scholz R, Maier-Hauff K et al (2006) The effect of thermotherapy using magnetic nanoparticles on rat malignant glioma. J Neurooncol 78:7-14

6. Visaria RK, Griffin RJ, Williams BW et al (2006) Enhancement of tumor thermal therapy using gold nanoparticle-assisted tumor necrosis factor- $\alpha$ delivery. Mol Cancer Ther 5:1014-1020

7. Johannsen M, Gneveckow U, Thiesen B et al (2007) Thermotherapy of prostate cancer using magnetic nanoparticles: feasibility, imaging, and three-dimensional temperature distribution. Eur Urol 52:1653-1662

8. Wilhelm C, Fortin J-P, Gazeau F (2007) Tumour cell toxicity of intracellular hyperthermia mediated by magnetic nanoparticles. J Nanosci Nanotechnol 7:2933-2937

9. Kikumori T, Kobayashi T, Sawaki M, Imai T (2009) Anti-cancer effect of hyperthermia on breast cancer by magnetite nanoparticleloaded anti-HER2 immunoliposomes. Breast Cancer Res Treat 113:435-441

10. Steeves RA (1992) Hyperthermia in cancer therapy: where are we today and where are we going? Bull N Y Acad Med 68:341

11. Asiaei S, Darvishi V, Davari MH et al (2019) Thermophoretic isolation of circulating tumor cells, numerical simulation and design of a microfluidic chip. $\mathrm{J}$ Therm Anal Calorim 137:831-839

12. Hobiny A, Abbas I (2021) Analytical solutions of fractional bioheat model in a spherical tissue. Mech Based Des Struct Mach 49:430-439

13. Alzahrani FS, Abbas IA (2019) Analytical estimations of temperature in a living tissue generated by laser irradiation using experimental data. J Therm Biol 85:102421

14. Ghanmi A, Abbas IA (2019) An analytical study on the fractional transient heating within the skin tissue during the thermal therapy. J Therm Biol 82:229-233

15. Phillips JL (2005) A Topical Review of Magnetic Fluid Hyperthermia. Am Inst Chem Eng Conf 14-18

16. Sun X, Song K, Wang S, Sun K (2021) Characterization of Magnetite/Iodized Oil Magnetic Fluid for Embolization and Hyperthermia Application. J Supercond Nov Magn 1-12

17. Vijayakanth V, Vinodhini V, Aparna A et al (2021) Synthesis and magnetic hyperthermia properties of zwitterionic dopamine sulfonate ligated magnesium ferrite and zinc ferrite nanoparticles. J Mater Sci Mater Electron 1-14

18. Usov NA, Nesmeyanov MS, Gubanova EM, Epshtein NB (2019) Heating ability of magnetic nanoparticles with cubic and combined anisotropy. Beilstein J Nanotechnol 10:305-314

19. Mejías R, Hernández Flores P, Talelli M et al (2018) CellPromoted Nanoparticle Aggregation Decreases NanoparticleInduced Hyperthermia under an Alternating Magnetic Field Independently of Nanoparticle Coating, Core Size, and Subcellular Localization. ACS Appl Mater Interfaces 11:340-355

20. Dabbagh A, Hedayatnasab Z, Karimian H et al (2018) Polyethylene glycol-coated porous magnetic nanoparticles for targeted delivery of chemotherapeutics under magnetic hyperthermia condition. Int J Hyperth 1-11

21. de Mello LB, Varanda LC, Sigoli FA, Mazali IO (2019) Coprecipitation synthesis of ( $\mathrm{Zn}-\mathrm{Mn})$-co-doped magnetite nanoparticles and their application in magnetic hyperthermia. J Alloys Compd 779:698-705

22. Fabris F, Lima E, De Biasi E et al (2019) Controlling the dominant magnetic relaxation mechanisms for magnetic hyperthermia in bimagnetic core-shell nanoparticles. Nanoscale

23. Engelmann UM, Buhl EM, Draack S et al (2018) Magnetic Relaxation of Agglomerated and Immobilized Iron Oxide Nanoparticles 
for Hyperthermia and Imaging Applications. IEEE Magn Lett 9:1-5

24. Mai BT, Balakrishnan PB, Barthel MJ et al (2019) ThermoResponsive Iron Oxide Nanocubes for an Effective Clinical Translation of Magnetic Hyperthermia and Heat-Mediated Chemotherapy. ACS Appl Mater Interfaces

25. Esmaeili E, Khalili M, Sohi AN et al (2018) Dendrimer functionalized magnetic nanoparticles as a promising platform for localized hyperthermia and magnetic resonance imaging diagnosis. J Cell Physiol

26. Ota S, Matsugi Y, Nakamura T et al (2018) Effects of size and anisotropy of magnetic nanoparticles associated with dynamics of easy axis for magnetic particle imaging. J Magn Magn Mater

27. Salloum M, Ma R, Zhu L (2008) An in-vivo experimental study of temperature elevations in animal tissue during magnetic nanoparticle hyperthermia. Int J Hyperth 24:589-601. https://doi.org/ 10.1080/02656730802203377

28. Salloum M, Ma RH, Weeks D, Zhu L (2008) Controlling nanoparticle delivery in magnetic nanoparticle hyperthermia for cancer treatment: Experimental study in agarose gel. Int J Hyperth 24:337-345. https://doi.org/10.1080/02656730801907937

29. Chen MY, Lonser RR, Morrison PF et al (1999) Variables affecting convection-enhanced delivery to the striatum: a systematic examination of rate of infusion, cannula size, infusate concentration, and tissue-cannula sealing time. J Neurosurg 90:315-320

30. Javidi M, Heydari M, Karimi A et al (2014) Evaluation of the effects of injection velocity and different gel concentrations on nanoparticles in hyperthermia therapy. J Biomed Phys Eng 4:151

31. Kandala SK, Liapi E, Whitcomb LL et al (2018) Temperaturecontrolled power modulation compensates for heterogeneous nanoparticle distributions: a computational optimization analysis for magnetic hyperthermia. Int J Hyperth 1-15

32. Gu Q, Joglekar T, Bieberich C et al (2019) Nanoparticle Redistribution in PC3 Tumors Induced by Local Heating in Magnetic Nanoparticle Hyperthermia: In Vivo Experimental Study. J Heat Transfer 141:32402

33. Hobiny A, Abbas I (2019) Thermal response of cylindrical tissue induced by laser irradiation with experimental study. Int J Numer Methods Heat Fluid Flow

34. Hobiny AD, Abbas IA (2020) Nonlinear analysis of dual-phase lag bio-heat model in living tissues induced by laser irradiation. J Therm Stress 43:503-511

35. Hobiny AD, Abbas IA (2018) Theoretical analysis of thermal damages in skin tissue induced by intense moving heat source. Int J Heat Mass Transf 124:1011-1014

36. Alavijeh MS, Maghsoudpour A, Khayat M et al (2020) Distribution of "molybdenum disulfide/cobalt ferrite" nanocomposite in animal model of breast cancer, following injection via differential infusion flow rates. J Pharm Investig 50:583-592

37. Gonzales-Weimuller M, Zeisberger M, Krishnan KM (2009) Sizedependant heating rates of iron oxide nanoparticles for magnetic fluid hyperthermia. J Magn Magn Mater 321:1947-1950

38. Ku G, Fornage BD, Jin X et al (2005) Thermoacoustic and photoacoustic tomography of thick biological tissues toward breast imaging. Technol Cancer Res Treat 4:559-565

39. Hawkridge AM (2014) The chicken model of spontaneous ovarian cancer. PROTEOMICS-Clinical Appl 8:689-699

40. Stewart DC, Rubiano A, Dyson K, Simmons CS (2017) Mechanical characterization of human brain tumors from patients and comparison to potential surgical phantoms. PLoS One 12:e0177561

41. Darvishi V, Navidbakhsh M, Amanpour S (2018) Effects of temperature distribution in the tissue around the tumor on the quality of hyperthermia. In: 2018 25th Iranian Conference on Biomedical Engineering and 2018 3rd International Iranian Conference on Biomedical Engineering, ICBME 2018

42. Xu LX, Zhu L, Holmes KR (1998) Thermoregulation in the canine prostate during transurethral microwave hyperthermia, part II: blood flow response. Int J Hyperth 14:65-73

43. Rylander MN, Feng Y, Zhang Y et al (2006) Optimizing heat shock protein expression induced by prostate cancer laser therapy through predictive computational models. J Biomed Opt 11:41113

44. Mcintosh RL, Anderson V (2010) A comprehensive tissue properties database provided for the thermal assessment of a human at rest. Biophys Rev Lett 5:129-151

45. Pennes HH (1998) Analysis of tissue and arterial blood temperatures in the resting human forearm. J Appl Physiol 85:5-34

46. Chang IA, Nguyen UD (2004) Thermal modeling of lesion growth with radiofrequency ablation devices. Biomed Eng Online 3:27

47. Darvishi V, Darvishi S, Bahrami-Bavani M et al (2021) Centrifugal isolation of SARS-CoV-2: numerical simulation for purification of hospitals' air. Biomech Model Mechanobiol. https://doi. org/10.1007/s10237-021-01477-x

48. Lin C-T, Liu K-C (2009) Estimation for the heating effect of magnetic nanoparticles in perfused tissues. Int Commun Heat Mass Transf 36:241-244

49. Attaluri A, Manuchehrabadi N, Dechaumphai A, Ma R, Zhu L (2011) Quantification of Nanostructure Distribution in Tissue Using MicroCT Imaging. ASME 2011 Summer Bioeng Conf SBC2011 1-2

Publisher's Note Springer Nature remains neutral with regard to jurisdictional claims in published maps and institutional affiliations. 